\title{
Cell biologist's perspective: Frontiers in the Development of PROTAC-HDAC degraders.
}

Alex Sobko, $\mathrm{PhD}$ *

*8762728, Ofakim, Israel; sobkosasha@ gmail.com. Previously at: The Department of Molecular Cell Biology, The Weizmann Institute of Science, Rehovot 76100, Israel. 


\section{Abstract}

This "Minireview and Perspective" article describes histone deacetylases (HDACs), as promising specific molecular targets to treat a variety of disease states by downregulating the expression of associated proteins with the use of a new generation of bioengineered compounds called protein targeting chimeras (PROTACs). We present the classification of HDACs, discuss their functions as key epigenetic regulators of gene expression, describe their roles in the biology of aging, describe histone- and nonhistone substrates of HDACs and their functions, and briefly introduce the concept of histonemodifying multiprotein complexes. Insight into the biological functions of specific HDACs comes from genetic knockout studies of individual genes encoding deacetylases. Initially discovered and newly developed HDAC inhibitors are powerful tools to investigate the functions of HDACs in cells and organs, that have also been successfully used in numerous preclinical and clinical studies, as promising drug candidates. We focus on the molecular and cellular mechanisms of their action, and introduce PROTACs, which are bivalent degrader molecules that have been recently developed to target HDACs. We then discuss recent studies focused on designing and testing several classes of selective and nonselective HDAC degraders in terms of their molecular and cellular mechanisms of action. Finally, we present open questions and new perspectives in developing the next generation of HDAC-degraders. 


\section{Contents:}

HDACs

Classification

Epigenetic regulators

HDACs and epigenetics of aging

Nonhistone substrates of HDACs

Histone modifying multiprotein complexes

Insight from knockout studies

HDAC inhibitors

Molecular and cellular mechanisms of action of HDAC inhibitors

Molecular mechanism of action

Cellular mechanism of action

Preclinical and clinical use of HDAC inhibitors

PROTACs: bivalent protein degraders

Class I HDAC degraders

HDAC6 degraders

PROTACs targeting NAD+-dependent histone deacetylases

Nonselective HDAC degraders

Histone methyltransferase-specific PROTACs

HDAC PROTACs: molecular mechanisms

HDAC PROTACs: cellular mechanisms

Design and development of PROTAC-mediated HDAC degradation

Testing HDAC degraders

Key questions and perspectives in developing HDACdegraders 


\section{HDACs. Classification}

The human histone deacetylase (HDAC) family consists of 18 enzymes, 11 of which contain a divalent zinc cation in the catalytic site, and the remaining seven are sirtuins with NAD+dependent activity.

HDACs can be further classified into four classes: class I (HDAC 1, 2, 3, and 8), class IIa (HDAC 4, 5, 7, and 9), class IIb (HDAC 6 and 10), class IV (HDAC11), and class III (sirtuins).

\section{Epigenetic regulators}

HDACs deacetylate lysine residues in the N-termini of $\mathrm{H} 3 / \mathrm{H} 4$ histones (e.g., H3K9ac, and H3K27ac). There is dynamic regulation of histone acetylation/deacetylation by histone deacetylases and histone acetyltransferases (HATs), which that counteract each other. HDACs function on the histones surrounding nucleosomes, and cause compact chromatin conformation. Compact chromatin is not accessible to RNA polymerase or, activators of gene expression, such as transcription factors and enhancers. Compaction of chromatin results in suppression of transcription of target genes.

In contrast to HDACs, HATs are recruited to activator complexes, and acetylate histones, leading to open chromatin conformation and activation of transcription by RNA polymerase (I/II). 


\section{HDACs and Epigenetics of Aging}

HDACs function on heterochromatin, maintaining it in an inactive, inert state. NAD-activated deacetylase Sir2, the master regulator of longevity in yeast, deacetylates the $\mathrm{N}$ termini of histones, leading to an inactive heterochromatin state and gene silencing. Loss of Sir2 shortens replicative lifespan, while overexpression and small molecule Sir2 activators extend lifespan (reviewed by Sen et al., 2016).

Studies in model organisms showed that caloric restriction and starvation are accompanied by increased HDAC activity. This suggests that in response to nutritional stress, global deacetylation may serve to protect cells and thereby influence aging processes (reviewed by Kenyon CJ, 2010, Morris BJ et al., 2019). In yeast, starvation is mimicked by rapamycin (inhibitor of TORC1 complex) treatment that displaces RNA polymerase I (pol I) from rDNA loci, thus inhibiting transcription at those sites. rDNA silencing is facilitated by increased binding of the Rpd3-Sin3 HDAC complex $\operatorname{Rpd} 3(\mathrm{~L}),-$ and deacetylation of $\mathrm{H} 4 \mathrm{~K} 5 / \mathrm{K} 12$ residues. These repressive chromatin effects of rapamycin serve to lower transcription of the protein synthesis apparatus, ultimately reducing growth (reviewed by Sen et al., 2016).

Epigenetic transgenerational effects of histone deacetylation are under active investigation. In females, the inactive $X$ chromosome has underacetylated histones, while the other $\mathrm{X}$ chromosome is active, with many histones being acetylated. In males, the status of Y chromosome histone acetylation needs to be further clarified. Determining if HDAC inhibitors cause increased histone acetylation and activation of genes in the inactive $\mathrm{X}$ chromosome is of interest. The possible effects of 
HDAC inhibitors on gene expression on the male $\mathrm{Y}$ chromosome are also worth to study.

\section{Studying nonhistone substrates of HDACs}

Certain HDACs function outside the nucleus, deacetylating nonhistone substrate proteins. For example, HDAC6 deacetylates the heat-shock protein HSP90 and components of the microtubule network, including $\alpha$-tubulin and $\beta$-tubulin (Jia Tong Loh, I-hsin Su, 2016), (Ningning Liu et al., 2015).

These activities of HDACs point to important functions of HDAC-regulated microtubules and cytoskeleton remodeling in cell division, cell migration and chaperone signaling (HSP90) during stress responses.

Histone acetylation was shown to be regulated by cell cycle progression (Krebs JE et al., 1999), which could be a reciprocal interaction, because HDAC inhibitors activate tumor suppressor genes and cause cell cycle arrest.

Nonenzymatic functions of HDACs are not so well understood and may include functions in scaffolding large complexes. Thus, HDACs interact with numerous transcription factors and may regulate their activities (Morris BJ et al., 2019). These nonenzymatic functions of HDACs can be investigated by HDAC degraders (see below), that do not affect HDAC activity, but downregulate HDAC protein levels. 


\section{Histone modifying multiprotein complexes}

Typically, canonical histone-modifying complexes consist of (1) targeting subunits that interact with DNA-binding proteins;

(2) HATs or HDACs; and (3) effector subunits acting on chromatin.

Thus, an HDAC is recruited and becomes a component of the corepressor complex responsible for the repression of transcription. This corepressor complex consists of (1) a DNA binding subunit, (2) a corepressor, and (3) an HDAC.

Important examples of the components of HDAC-co-repressor complexes include: MyoD; Mad-Max heterodimer; SMRT; MeCP2 (mutated in Rett syndrome); and polycomb repressive complex II ("enhancer of zeste" EZH2, histone-binding protein, HDAC). An interesting example of gene regulation by HDACs in yeast is the regulation of the GAL1/10 system. This response involves the methylation of histones (H3K36me2me3) of target genes (GAL1 and, GAL10), leading to the recruitment of HDACs and histone deacetylation resulting in the activation of the expression of these genes.

\section{Insight from knockout studies}

The review article by Daniel Alencar Rodrigues, and colleagues (Rodrigues, 2022), presents and discusses very important information on the translational significance of examining the phenotypes of mice bearing knockouts of individual HDAC genes. These data suggest that HDAC inhibitors should be developed and tested with caution and with their safety being rigorously tested, due to the important roles played by individual HDACs in embryonic and postnatal 
development. This information is very important for drug development because of the possible predicted teratogenic effects of HDAC inhibitors. It is possible that HDAC inhibitors targeting certain HDACs may need to be excluded from use in pregnant women and those who may become pregnant.

It is also important to consider immunomodulation, metabolism and other functions of individual HDAC genes in more detail. For example, knockout studies point to important potential targets of HDAC6 downregulation, such as targets in Alzheimer's disease and other neurodegenerative diseases, inflammation and cancer metastasis, due to the nonhistone functions of HDAC6, such as its effects on cell migration via increased hyperacetylation of $\beta$-tubulin (reviewed by Jia Tong Loh, I-hsin Su, 2016).

\section{HDAC inhibitors}

Important insights into the functions of HDACs come from the use of HDAC inhibitors. In initial studies, two major compounds with HDAC inhibitory activity were discovered and tested: (1) trichostatin A (TSA), and (2) butyric acid. The application of inhibitors in yeast leads to accumulation of histone-acetylated nucleosomes, and activation of gene expression. They also cause acetylation of centromeric heterochromatin. The use of inhibitors may also cause epigenetic (possibly, transgenerational) effects, so that the acetylated state of histones is passed to the next generation of the cells, following cell division. To the best of my knowledge, the possible epigenetic transgenerational effects of HDAC inhibitors on human beings are unknown. 


\section{Molecular and cellular mechanisms of action of HDAC inhibitors}

\section{Molecular mechanism of action}

All HDAC inhibitors are thought to function as histone acetylation/deacetylation-specific epigenetic regulators. They also affect nonhistone substrates of HDACs outside the nucleus (such as tubulins and heat shock proteins). It is also possible that HDAC-specific PROTACs affect the noncatalytic functions of HDACs, which are not well-understood. The therapeutic (epigenetic) mechanism of HDAC inhibitors is thought to be significantly enhanced when they are used in combination with demethylating agents, such as 5-aza-2deoxycitidine (aza-citidine). This mechanism is based on the finding that HDACs are recruited to the proteins bound to methylated cytosines, so preventing both binding of the complex to the target genes and inhibiting HDAC activity is thought to enhance the effect of HDAC inhibitors on the expression of target genes.

\section{Cellular mechanism of action}

The cellular mechanism of action of HDACs is being actively investigated. The observed cellular effects include the arrest of cell differentiation, regulation of cell cycle progression, cellular senescence, and apoptosis. At the molecular level, it is accompanied by the accumulation of reactive oxygen species (ROS), activation of the DNA damage response (DDR), and disruption of chaperone signaling. It is thought that histone acetylation/deacetylation affects the expression of tumorsuppressor genes, and the activation of tumor-suppressor genes leads to cell cycle arrest and apoptosis. 
HDACs participate in DNA repair, and their depletion by RNAi or specific inhibitors leads to increased YH2AX expression and heightened DDR. They modulate the response to therapyinduced genotoxic stress and therapy-induced senescence (TIS). Vorinostat, a panhistone deacetylase (HDAC) inhibitor, is a potential TIS agent that elicits DDR. Vorinostat alters the normal chromatin structure. HDAC inhibitors might be promising senolytic drug candidates with many possible applications.

\section{Preclinical and clinical use of HDAC inhibitors}

Several HDAC inhibitors have been approved for clinical use, and several new inhibitor candidates are currently in preclinical and clinical trials for numerous pathologies, such as cancers, immune disorders and neurological disorders. They may be involved in modulating inflammation, autoimmune disorders and asthma. As of 2020, the US Food and Drug Administration (FDA) had approved five HDAC inhibitors for the treatment of T-cell lymphomas, multiple myeloma, and acute myeloid leukemia (Ho TC et al., 2020). These compounds are also in trials for the treatment of solid cancers (prostate cancer, breast cancer, lung cancer, head and neck cancer, NSCLC, and HNSCC). The potential use of HDAC inhibitors in Alzheimer's disease and other neurodegenerative diseases has been reviewed recently (Rodrigues et al., 2020).

There are nonselective pan-HDAC inhibitors and selective inhibitors (e.g., HDAC3-, HDAC4-, and HDAC6-selective). The pan-HDAC inhibitors vorinostat (suberanilohydroxamic acid, or SAHA) and dacinostat target the class I and IIb HDACs 
$1,2,3,6,8$, and 10. TMP269 and NVS-HD1 are targeting target the class IIa HDACs 4, 5, 7, and 9.

The most important application of HDAC inhibitors is the treatment of cancers. Thus, vorinostat, romidepsin, and belinostat are used in cutaneous T-cell lymphoma (CTCL), peripheral T-cell lymphoma (PTL), and acute myeloid leukemia. Panobinostat is used for the treatment of multiple myeloma. HDAC inhibitors are also being tested in solid cancers (prostate cancer, lung cancer, and head and neck cancer). Global Aurora kinase inhibition (AMG900) in combination with HDAC inhibition resulted in the upregulation of senescence features in prostate cancer models (Paller et al., 2014). Panobinostat, was investigated for its potential to clear lung, cancer and head and neck cancer cells induced into senescence by cisplatin or paclitaxel in vitro. Chemotherapyinduced senescent tumor cells (NSCLC and HNSCC) were more vulnerable to the toxic effects of panobinostat in comparison to their proliferating counterparts (Samaraweera $\mathrm{L}$ et al., 2017). Interestingly, the senolytic activity of panobinostat was also linked to its effect on Bcl-xL expression levels (reviewed by Saleh T et al., 2020).

HDAC inhibitors are also tested or used in certain autoimmune diseases and asthma. It is also important to consider the effects of histone acetylation/deacetylation in immune responses to infection. The gram-negative bacterial endotoxin LPS, which causes inflammation and the release of inflammatory cytokines, such as TNF-alpha, increases the activities of HATs and suppresses the activities of HDACs, overall increasing histone acetylation and the activation of the genes responsible for the anti-inflammatory immune response. In contrast, steroids, such 
as glucocorticoids, act through HDACs (e.g., HDAC2) to suppress the expression of proinflammatory genes.

Preclinical trials of HDAC-specific senolytics are also being conducted. Modulators of therapy-induced senescence (agents that induce accelerated TIS) include: sodium butyrate (a cClass I and II HDAC inhibitor), trichostatin A (a class I and II HDAC inhibitor), Ms-275 (a class I HDAC inhibitor), vorinostat, or SAHA (a class I and II HDAC inhibitor), panobinostat, or Lbh589 (a class I and II HDAC inhibitor), 4-phenylbutyric acid (a class I and IIa HDAC inhibitor), valproic acid (a class I and IIa HDAC inhibitor) (Faheem et al., 2020).

Combined treatment with an aurora kinase inhibitor and HDAC inhibitor, induced senescence in prostate cancer models. Lung and, head and neck cancer cells (NSCLCs and, HNSCCs) induced into senescence by cisplatin or paclitaxel, are cleared by panobinostat (reviewed by Saleh T et al., 2020).

\section{PROTACs: bivalent protein degraders}

In this review, we do not provide an initial or comprehensive introduction into bivalent protein targeting chimeras (PROTACs). These compounds have been recently reviewed in the leading journals. They are subject to the recent publications and presentations at recent conferences focused on targeted protein degradation with the participation of leading academic and industry labs worldwide.

Current PROTAC clinical trials focus on compounds targeting androgen receptors (to develop drugs against prostate cancer) and estrogen receptors (to develop drugs against breast cancer). Previously, bromodomain proteins (epigenetic readers 
targeting acetylated lysines) have been actively used to develop several PROTAC molecules.

Recent studies of HDAC-PROTACs test either individual molecules or libraries of compounds in single or multiple rounds of selection.

HDAC degraders recently developed include class I HDAC degraders, HDAC6 degraders, NAD+-activated SIRT degraders and nonselective HDAC degraders.

We would like to briefly mention several recent examples of newly developed HDAC degraders.

\section{Class I HDAC degraders}

An HDAC1-specific degrader based on the dTAG system was designed: degradation of a fusion protein between F36V FKBP-ILigand and an IMiD derivative (Nabet et al., 2018, Burslem and Crews, 2020).

An HDAC3-specific degrader was reported by Xiao Y. et al., Chem Comm, 2020. A compound called XZ9002 is a PROTAC based on either CRBN or VHL, and it was tested with in breast cancer cell lines for HDAC3 degradation, histone acetylation, and cell proliferation. This compound may target breast cancer metastasis. Future work will examine the antimetastatic effects of XZ9002 in cellular and animal models.

\section{HDAC6 degraders}

In 2018, Yang K et al., 2018 published one of the first original reports of the development of an HDAC6 degrader. Relatively 
nonselective HDAC inhibitors were used in cell-based assays to select for more HDAC6-specific compounds. For instance, one such compound "compound 18" increased acetylation of histone H3. HDAC6 inhibitors might modulate the acetylation of nonhistone proteins, such as tubulin and HSP90. Future studies will focus on how HDAC6-PROTACs affect the acetylation of these nonhistone proteins and their cellular functions.

The initial work of that group was followed up by testing the library of CRBN ligands in cell-based (target-engagement) binding assays with the read-out for HDAC6 degradation (Western blot and mass spectrometry) (Yang K et al., 2020). The advantage of the system is that it is based on the analysis of endogenous target substrate proteins.

In 2019, Wu H et al. also reported a selective HDAC6 degrader (Nexturostat-CRBN), targeting histones and cytoplasmic substrates of HDAC6 (tubulin and HSP90) that can be candidates for the treatment of multiple myeloma.

Tim Keuler and colleagues recently reported a new generation of HDAC6 degraders with optimized chemical design (Chem. Commun., 2022, 58, 11087-11090).

\section{PROTACs targeting NAD+-dependent histone deacetylases}

In 2018, Schiedel $M$ et al. developed SIRT2-specific compounds with so-called "SirReals", - derivatives of thalidamide, that recruit CRBN E3 ligase and tested their activities in HeLa cells. 


\section{Nonselective HDAC degraders}

A landmark study was recently published by Fischer lab (Xiong et al., 2021), reporting a chemo-proteomics platform to test a panhistone deacetylase (HDAC) degrader library. They carefully examined and discussed all important factors for developing new HDAC degraders and proposed a molecular mechanism to account for the TPD of multiprotein (repressor) HDAC-containing complexes.

An interesting new approach applied to pan-HDAC inhibitors was developed by Friedman Ohana et al., 2021. They developed a "Photo-affinity tag" system, in which the photoaffinity tag binds the target protein. This binding was interfered by the use of a pan-HDAC inhibitor in the protein-inhibitor interaction (binding) assay. The photoaffinity tag binds the target protein upon cell treatment followed by UV photocrosslinking, lysis, capture/enrichment/release with Halo-Tag beads, and chemical cleavage. BRET was used as a read-out assay.

\section{Histone methyltransferase-specific PROTACs}

Preventing histone methylation is thought to affect "a code of histone modifications" and enhance the effects of HDAC inhibition. There is a new generation of patented histonemodifier "non-E3 ligase"-based bifunctional degraders recruiting the ubiquitin receptor, which targets the substrate for degradation by the proteasome. One example of this new type of histone modifier is the "DOT1L" degrader recently patented by DFCI and specific for histone (H3K79me1me2me3) methyltransferase. It is a bifunctional "non-E3 ligase"-based degrader that links the DOT1L binder and RA190 binder of 
ubiquitin receptor RPN13, targeting DOT1L to degradation by the proteasome (discussed by "Ciulli lab TPD Journal Club", School of Life Sciences, University of Dundee, UK).

\section{HDAC PROTACs: molecular mechanisms}

Considering the molecular mechanism of TPD by HDAC degraders, they have been shown to bind and target either free HDAC proteins or HDAC-containing multiprotein complexes. Thus, "collateral" degradation of other proteins within HDACcontaining complexes has been demonstrated. This may lead to the loss of chromatin repressive complexes. Xiong et al., 2021 proposed two possible mechanisms to account for the effect of degraders on collateral degradation:

- direct ubiquitination and subsequent degradation of HDAC-binding partners that are located in proximity to E3 ligase;

- ubiquitination and subsequent degradation of HDAC proteins that leads to destabilization of the complex, which is then degraded by the proteasome.

\section{HDAC PROTACs: cellular mechanisms}

The cellular effects of HDAC PROTACs are being actively investigated. They might include: target cell-, tissue-, and organ-specific effects, cell differentiation arrest, and reciprocal regulation of cell cycle progression, cellular senescence, and apoptosis. At the molecular level, HDAC-PROTACs may affect the accumulation of reactive oxygen species (ROS) and the DNA damage response (DDR), and disrupt chaperone signaling. It is thought that histone acetylation/deacetylation regulates the expression of tumor- suppressor genes. The activation of tumor- suppressor genes leads to cell cycle arrest 
and apoptosis. It is important to mention modulation of DNA repair, YH2AX expression, and DDR. They might modulate the cellular response to therapy-induced genotoxic stress and therapy-induced senescence (TIS). Based on their mechanism of action, HDAC-PROTACs are promising drug candidates with many possible applications (for diseases, in which HDAC inhibitors have been shown to be effective).

\section{Design and Development of PROTAC-mediated HDAC Degradation}

HDAC-PROTACs are designed based on

(I) ligands targeting HDAC;

(II) E3 ligases: (CRBN, VHL, and IAP);

(III) molecular scaffolds; and

(IV) linkers (of various chemical structures, lengths and attachment points).

The key criteria for developing HDAC-PROTACs include

(1) the molecules tested (individual molecules versus libraries);

(2) "top-down" (targeting families) versus "bottom-up" approaches;

(3) the ligands, E3 ligases, scaffolds and linkers tested; and

(4) the selectivity of the degraders (based on Pan-HDAC versus selective inhibitors).

Typically, several assays have been employed to test degrader activity: (A) measuring levels of target proteins by Western blot (band intensity, "isoform-specific shift"), fluorescent reporterbased assays, quantitative proteomics (measuring HDAC protein half-life, and the global effects at the proteome level); (B) HDAC ubiquitination assays; and (C) HDAC enzymatic activity assays (in vivo and in vitro) - for example, 
fluorescence activity assays sensitive to trypsin cleavage, measuring the inhibitory effect of the compound on HDAC activity. It is important to include appropriate controls in these assays: inactive E3 ligase (no effect on TPD); proteasome inhibitor(s) (negative effect on TPD); and specific effective HDAC inhibitors (the control for HDAC activity).

It is also important to examine the effects of HDAC degradation on the expression of other proteins -, including significantly upregulated and downregulated proteins identified in chemoproteomics experiments.

Another possible effect that can be tested is phenotype rescue of the phenotype (ex. age-dependent regulation of the expression of certain proteins) by the deletion (or downregulation) of specific HDACs (see, for example, the chapter on HDAC knockouts).

Recently, new methods have been developed to study HDACPROTACs, such as global activity-based protein profiling (ABPP) (Cravatt group, Scripps Research Institute, La Jolla, CA.) This functional proteomics "in-gel" platform is based on the use of "active site"-directed probes in whole-proteome profiling and competitive assays with candidate degrader molecules. Such probes label active enzymes (for example, specific PTMs) but not inactive, inhibitor-bound enzymes. Therefore, competitive ABPP can be used for the discovery of selective inhibitors of enzymatic activity, including HDACs.

The steps of ABPP include:

1) Labeling of the active, but not inactive enzyme; 
2) In-gel fluorescence scanning and analysis of enzymatic activity (normal versus disease, control versus inhibitor, or control versus degrader); and

3) Identification of labeled enzymes by affinity purification/mass spectrometry.

ABPP is being tested with HDAC-specific probes in competitive assays. Treatment can be performed with the library of the candidate molecules -, inhibitors/PROTACs (treated versus nontreated), followed by "in-gel" analysis. Typically, this kind of assay is applicable to in vitro cancer cell models, in vivo cancer animal models, and primary tumor specimens. There is also a tag-free version of ABPP applicable to live cells and animals, i.e., active in vivo (Zhang X et al., 2019), (Salisbury, Cravatt, 2007), (Salisbury, Cravatt, 2008).

\section{Testing HDAC degraders}

Each degrader molecule has to be tested for:

- antiproliferative activity and other relevant cell-based assays

- selectivity and promiscuity of the compound toward HDACs

- off-target degradation

- $\quad$ toxicity, adverse effects

- $\quad$ cell line specificity (cell-based assays).

- time-course, (determining the peak of degradation)

- $\quad$ possible cell cycle-dependent effects (Krebs JE et al.et al, 1999).

The following effects were examined: specific scaffolds; E3 ligase activity (including ligase-inactive control); and linker 
chemistry (varying the length of the linker, attachment sites, etc.). These testing criteria were thoroughly discussed in the recent article by Xiong et al., 2021.

\section{Key open questions and perspectives in developing HDAC- degraders}

One potential future direction of research on HDAC degraders is to select them based on the expression of the native E3 ligase in the target organ/tissue, to enable a cell-type specific TPD for more focused therapeutic effects. In principle, it is possible to use the data of the protein atlas, cancer atlas and other databases of protein expression to select E3 ligase candidates that are sufficiently expressed in the target organ/tissue.

Although nearly all compounds work through the ubiquitinproteasomal pathway (sensitive to proteasome inhibitors), there is also a new generation of patented histone-modifier "non-E3 ligase"-based bifunctional degraders that recruiting the ubiquitin receptors (such as RPN13), to target the substrate to the for degradation by the proteasome (discussed by "Ciulli lab TPD Journal Club", School of Life Sciences, University of Dundee, UK).

Delivery of the therapeutic compound to the target tissue/organ and permeability of the cell membranes to a given compound are very important factors that influence their therapeutic value. This topic has been discussed in recent articles.

Other questions for discussion and future development: 
- Does particular degrader target HDACs only in the nucleus or also in other cell compartments (see for example, Zhang $\mathrm{X}$ et al., 2019)?

- Is it feasible to design cell compartment-specific degraders?

- What is the composition of multiprotein complexes that contain specific target HDACs?

- Does the composition of the multiprotein complex change over time?

- How specific or promiscuous is the degrader-HDAC binding?

- Emphasizing the importance of diversity in designing the components of the degrader (variety of scaffold, linker, and E3 ligase candidates).

Due to the mechanism of action of HDACs, it is also worth mentioning PROTACs targeting other (non-HDAC) histone modifying enzymes, such as DOT1L, a degrader, mentioned above. The therapeutic (epigenetic) mechanism of HDAC inhibitors is thought to be significantly enhanced by use in combination with demethylating agents, such as 5-aza-2deoxycitidine (aza-citidine). This mechanism is based on the finding that HDACs are recruited to the proteins bound to methylated cytosines, so preventing both binding of the complex to the target genes and inhibiting HDAC activity is thought to enhance the effect of HDAC inhibitors on the expression of target genes. 
Since HDAC inhibitors have been successfully combined with aurora kinase inhibitors and other chemotherapeutic drugs that induce cellular senescence, in future trials, it will be possible to combine these compounds with HDAC-targeting PROTACs.

Recently, the novel generation of PROTAC compounds targeting plasma membrane integral proteins, based on the use of the plasma membrane-bound E3 ligase-coupled antibodies was reported (Marei H. et al., 2022). This promising strategy needs to be further elaborated and optimized to target cytosolic and nuclear HDAC degraders. 


\section{Literature Cited}

\section{Books:}

Epigenetics, Second Edition, Edited by C. David Allis et al, CSHL Press. (C) 2015. Chapter 4. Writers and Readers of Histone Acetylation: Structure, Mechanism, and Inhibition. Ronen Marmorstein, Ming-Ming Zhou. Chapter 5. Erasers of Histone Acetylation: The Histone Deacetylase Enzymes. Edward Seto, Minoru Yoshida. Chapter 34. Epigenetic Determinants of Cancer. Stephen B. Baylin, Peter A. Jones. Chapter 35. Histone Modifications and Cancer. James E. Audia Robert M. Campbell.

Introduction to Epigenetics. Learning Materials in Biosciences. Chapter: Epigenetics and Cancer. Histone Acetylation and Deacetylation in Cancer. By Renato Paro, Ueli Grossniklaus, Raffaella Santoro and Anton Wutz. (C) 2021 Springer Nature Switzerland AG; Open Access book is licensed under the terms of the Creative Commons Attribution 4.0 International License.

Lewin's Genes XII. Krebs, Jocelyn E., Goldstein, Elliott S., Kilpatrick, Stephen T. Jones \& Bartlett Learning, 2018.

Ageless Quest: One Scientist's Search for the Genes That Prolong Youth. Leonard Guarente. CSHL Press, 2002.

Lifespan: Why We Age-and Why We Don't Have To. David A. Sinclair PhD, Matthew D. LaPlante. 2019.

Harrison's Principles of Internal Medicine, 20-th Edition. (C) 2018 by McGraw-Hill. 


\section{Review articles:}

Burslem GM and Crews CM. Proteolysis-Targeting Chimeras as Therapeutics and Tools for Biological Discovery. Cell (2020).

Ciulli A. and Trainor N. A beginner's guide to PROTACs and targeted protein degradation. October 2021 (C) The Authors. Portland Press Limited under the Creative Commons Attribution License 4.0 (CC BY-NC-ND).

Faheem et al. Convergence of therapy-induced senescence (TIS) and EMT in multistep carcinogenesis: current opinions and emerging perspectives. Cell Death Discovery (2020) 6:51.

Ho TC et al., Thirty Years of HDAC Inhibitors: 2020 Insight and Hindsight. J. Med. Chem., 2020, 63, 12460-12484.

Kenyon CJ. The genetics of ageing. Nature, 2010, 464, 504. Cell 166, 2016.

Loh JT, Su I. Post-translational modification-regulated leukocyte adhesion and migration. Oncotarget, 2016, 7(24), 37347-37360.

Morris BJ et al, Genetic and epigenetic regulation of human aging and longevity. Biochim Biophys Acta, 2019, Mol Basis Dis, 1865(7), 1718-1744.

Rodrigues DA, Pedro de S. M. Pinheiro, Fernanda S. Sagrillo, Maria L. Bolognesi, Carlos A. M. Fraga. Histone deacetylases as targets for the treatment of neurodegenerative disorders: Challenges and future opportunities. Med Res Rev. 2020; 1-35. 
Rodrigues DA, Roe A, Griffith D, Chonghaile TN. Advances in the Design and Development of PROTAC-mediated HDAC Degradation. Curr Top Med Chem. 2022 Mar 4;22(5):408-424. Schreiber SL. The Rise of Molecular Glues. Cell 2021, 184.

Sen P, Parisha P. Shah, Raffaella Nativio, and Shelley L. Berger. Epigenetic Mechanisms of Longevity and Aging. Cell 2016, 166, 822-839.

Severini L, Bufalieri F, Infante P, Di Marcotullio L. Proteolysis-Targeting Chimera (PROTAC): Is the Technology Looking at the Treatment of Brain Tumors? Front Cell Dev Biol. 2022 Feb 15; 10:854352.

Tareq S, Sarah Bloukh, Valerie J. Carpenter, Enas Alwohoush, Jomana Bakeer, Sarah Darwish, Belal Azab, and David A. Gewirtz. Therapy-Induced Senescence: An "Old" Friend Becomes the Enemy. Cancers, 2020, 12, no. 4: 822.

Verma R, Dane Mohl, and Raymond J. Deshaies. Harnessing the Power of Proteolysis for Targeted Protein Inactivation. Molecular Cell, 2020, 77.

Wu, T., Yoon, H., Xiong, Y. et al. Targeted protein degradation as a powerful research tool in basic biology and drug target discovery. Nat Struct Mol Biol 27, 605-614 (2020).

\section{Research Articles:}

Durbin AD, Wang T, Wimalasena VK, Zimmerman MW, Li D, Dharia NV, Mariani L, Shendy NAM, Nance S, Patel AG, Shao Y, Mundada M, Maxham L, Park PMC, Sigua LH, Morita K, 
Conway AS, Robichaud AL, Perez-Atayde AR, Bikowitz MJ, Quinn TR, Wiest O, Easton J, Schönbrunn E, Bulyk ML, Abraham BJ, Stegmaier K, Look AT, Qi J. EP300 Selectively Controls the Enhancer Landscape of MYCN-Amplified Neuroblastoma. Cancer Discov. 2022 Mar 1;12(3):730-751. doi: 10.1158/2159-8290.CD-21-0385.

Friedman Ohana R, Sergiy Levin, Robin Hurst, Michael M. Rosenblatt, Kristopher Zimmerman, Thomas Machleidt, Keith V. Wood, and Thomas A. Kirkland. Streamlined Target Deconvolution Approach Utilizing a Single Photoreactive Chloroalkane Capture Tag. ACS Chemical Biology 202116 (2), 404-413.

Keuler T. et al, Development of the first non-hydroxamate selective HDAC6 degraders. Chem. Commun., 2022,58, 11087-11090

Krebs JE et al, Cell cycle-regulated histone acetylation required for expression of the yeast HO gene. Genes Dev, 1999, 13, 1412-1421.

Liu N, Yun Xiong, Shanshan Li, Yiran Ren, Qianqian He, Siqi Gao, Jun Zhou, \& Wenqing Shui. New HDAC6-mediated deacetylation sites of tubulin in the mouse brain identified by quantitative mass spectrometry. Scientific Reports, 2015, 5:16869.

Marei, H., Tsai, WT.K., Kee, YS. et al. Antibody targeting of E3 ubiquitin ligases for receptor degradation. Nature 610, 182 189 (2022). 
Nabet, B., Roberts, J.M., Buckley, D.L. et al. The dTAG system for immediate and target-specific protein degradation. Nat Chem Biol, 2018, 14, 431-441.

Paller $\mathrm{CJ}$ et al, Combining the pan-aurora kinase inhibitor AMG 900 with histone deacetylase inhibitors enhances antitumor activity in prostate cancer. Cancer Med. 2014, 3, 1322-1335.

Salisbury CM, Cravatt BF, Activity-based probes for proteomic profiling of histone deacetylase complexes. PNAS, 2007, 104, 4, 1171-1176.

Salisbury CM, Cravatt BF, Optimization of Activity-Based Probes for Proteomic Profiling of Histone Deacetylase Complexes. J Am Chem Soc, 2008, 130, 7, 2184-2194.

Samaraweera, L., Adomako, A., Rodriguez-Gabin, A. et al. A Novel Indication for Panobinostat as a Senolytic Drug in NSCLC and HNSCC. Sci Rep, 2017, 7,1900.

Schiedel $\mathrm{M}$ et al, Chemically Induced Degradation of Sirtuin 2 (Sirt2) by a Proteolysis Targeting Chimera (PROTAC) Based on Sirtuin Rearranging Ligands (SirReals). J Med Chem, 2018, $61,2,482-491$.

$\mathrm{Wu} \mathrm{H}$ et al, Development of Multifunctional Histone Deacetylase 6 Degraders with Potent Antimyeloma Activity. J Med Chem, 2019.

Xiao Y. et al, Discovery of histone deacetylase 3 (HDAC3)specific PROTACs. Chem Comm, 2020. 
Xiong et al, Chemo-proteomics exploration of HDAC degradability by small molecule degraders. Cell Chemical Biology, 2021.

Yang K et al, Development of the first small molecule histone deacetylase 6 (HDAC6) degraders. Bioorg Med Chem Lett, 2018.

Yang K et al, A Cell-Based Target Engagement Assay for the Identification of Cereblon E3 Ubiquitin Ligase Ligands and Their Application in HDAC6 Degraders. Cell Chem Biology, 2020.

Zhang X, Crowley VM, Wucherpfennig TG et al, Electrophilic PROTACs that degrade nuclear proteins by engaging DCAF16. Nat Chem Biol, 2019, 15, 737-746.

Zhang X, Crowley VM, Wucherpfennig TG et al, Electrophilic PROTACs that degrade nuclear proteins by engaging DCAF16. Nat Chem Biol, 2019, 15, 737-746.

\section{Online Resource Cited:}

Ciulli Laboratory (School of Life Sciences, University of Dundee) Journal Club Targeted Protein Degradation and other literature highlights:

https://www.lifesci.dundee.ac.uk/groups/alessiociulli//publications/journal-club 


\section{Figures:}

Figure 1. Alterations of HATs and HDACs in cancers (Borrowed from: "Introduction to Epigenetics". Learning Materials in Biosciences. By Renato Paro, Ueli Grossniklaus, Raffaella Santoro and Anton Wutz, Springer, 2021; under the terms of the Creative Commons Attribution 4.0 International License).

Figure legend. Alterations in the acetylation/deacetylation balance can influence the expression of tumor suppressor genes and oncogenes, favoring the tumorigenic process. High expression or the abnormal recruitment of HDACs or the reduced expression or activity of HATs can lead to the repression of tumor suppressor genes. In contrast, mutation or low expression of HDACs or high expression, oncogenic fusion, or aberrant recruitment of HATs can lead to the activation of oncogenes.

Figure 2. Illustration of the concept of epigenetics. Author with his daughters in Southern California in 2002.

Figure legend. Photo of the author (Alex Sobko) wearing a sombrero hat, with two of his daughters during the celebrations of the Jewish holiday of Purim at Soille San Diego Hebrew Day School in 2002.

\section{Acknowledgments}

The author is grateful to the reviewers for their critical review of the manuscript. 